\title{
A Methodology for Introducing M\&V Adjustments during an Energy Retrofit Impact Assessment
}

\author{
Nikos Sakkas, Evangelos Kaltsis \\ Applied Industrial Technologies Ltd., Gerakas, Greece \\ Email: sakkas@apintech.com
}

Received 7 August 2014; revised 7 September 2014; accepted 13 September 2014

Copyright (C) 2014 by authors and Scientific Research Publishing Inc.

This work is licensed under the Creative Commons Attribution International License (CC BY). http://creativecommons.org/licenses/by/4.0/

(c) (i) Open Access

\begin{abstract}
The assessment of an energy retrofit necessarily requires an energy measurement campaign before (base year energy consumption) and after (post retrofit energy consumption) the retrofit. Only in this way is it possible to reach a safe conclusion, on the true retrofit impact. In addition, a number of adjustments are necessary to secure that the retrofit impact on energy consumption is effectively isolated, i.e., which we report on the true retrofit impact and not, for example, on external variations, such as a more mild winter. This paper introduces a conceptual framework for taking account, in the retrofit impact assessment, of three external parameters: weather, indoor comfort and space occupancy. The broader strategy behind this work is to develop a comprehensive methodology that would allow a cost efficient, fast and accurate assessment of energy retrofits in buildings. This would allow insight, on the investor side, as to the prudence of his investment and, and in this way, could help the proliferation of the practice of energy retrofits. The adjustment methodology, introduced here, is a first step in this direction.
\end{abstract}

\section{Keywords}

Energy Retrofit, Impact Assessment, External Parameter Adjustment, Occupancy, Operational Retrofit

\section{Introduction}

Building retrofits are an important activity in the construction industry. They may be initiated for many reasons, one of which is the reduction of energy consumption (energy retrofits). What remains a key barrier for a wider uptake of energy retrofits is the difficulty one encounters to measure and communicate their impact. As a result, 
the lack of data backed evidence on the retrofit impact does not allow the building owner to gain insight in his investment. Similarly, it does not allow the retrofit provider to fully understand his solution limitations and launch continuous improvement strategies.

According to IPMVP (International Performance Measurement and Verification Protocol), measuring energy savings should be based on the following general equation:

$$
\text { Energy Savings }=\text { Baseline (pre retrofit) Energy Use }- \text { Post Retrofit Energy Use } \pm \text { Adjustments }
$$

Adjustments are required to account for changes of external conditions. Weather and occupancy information are key external parameters, suggested for use in M\&V protocols [1] [2]. They will also be used in our approach. The uncertainty of such data has received significant attention in the literature [3]-[6]. Weather data are often taken from distant meteorological stations and might fail, in this way, to capture micro-climate conditions around the specific building. Occupancy presents important difficulties to measure. Several approaches have been proposed in the literature for assessing baseline as well as post retrofit energy use. Kissock [7] developed a regression methodology to measure retrofitting energy use in commercial buildings. Krarti et al. [8] utilized neural networks to estimate energy and demand savings from retrofits of commercial buildings. Dhar et al. [9] generalized the Fourier series approach to model hourly energy use in commercial buildings. In addition, in most practical cases, utility bill data are used because they are widely available and inexpensive to obtain and process. Reddy et al. [10] presented a formal baselining methodology at the whole building level based on monthly utility bills and took outdoor dry-bulb temperature as the only model regressor.

This research background reveals that adjustments are only one of the many difficulties that need to be overcome towards our end goal, i.e., an accurate, reliable and cost efficient assessment of the retrofit impact. However, even defining the baseline energy consumption, in a cost effective and practical way, may, in some cases, present significant difficulties. Let us imagine, for example, the case where a sub-part (e.g. a floor) of a building, equipped with a centralized HVAC system, is retrofitted. How can one define here the baseline consumption? This is not a trivial question if we wish to end up with a practical solution and avoid costly measurements of the many parameters contributing to it (e.g. air duct velocities). Obviously, scaling down building level data to our reduced retrofit scope (e.g. floor) would introduce large errors and can not be recommended.

Another critical issue for the viability of a retrofit assessment procedure is the duration of the campaign. Any scheme requiring more than a couple of weeks of monitoring before and after the retrofit would stand little chances for business uptake.

In this paper, we touch upon only one of the many issues that are related to this key issue of retrofit assessment. We discuss the issue of $\mathrm{M} \& \mathrm{~V}$ adjustments of parameters that may be different before and after the retrofit and may, because of this, affect our assessment. We propose a methodological framework to address three types of possible parameter variation: weather, indoor conditions and building occupancy. This is summarized in Figure 1.

\section{Methods}

We will define our baseline impact, prior to any adjustment, by means of Equation (2). This equation essentially calculates the change of energy consumption, of our study building space, before and after the retrofit, as a percentage of the former energy consumption. Energy consumed is measured in the real time, at an equal, before and after the retrofit, number of time instances $[\mathrm{N}]$.

$$
\text { Baseline Impact }=\left[\left[\sum_{i=1}^{N} E_{[\mathrm{pre}, \mathrm{i}]}\right]-\left[\sum_{i=1}^{N} E_{[\mathrm{post}, \mathrm{i}]}\right]\right] /\left[\sum_{i=1}^{N} E_{[\mathrm{pre}, \mathrm{i}]}\right]
$$

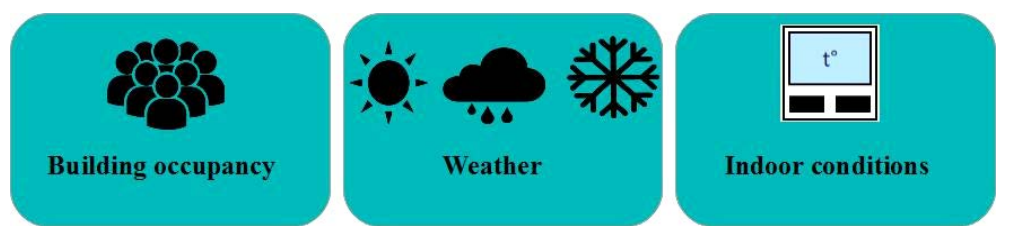

Figure 1. Weather, building occupancy and indoor conditions included as the $\mathrm{M} \& \mathrm{~V}$ adjustments to assess the true energy retrofit impact. 
pre, the pre-retrofit period, year or other.

post, the post-retrofit period, of a similar duration with the pre-retrofit one.

$\boldsymbol{i}$, a measurement instance.

$N$, the number of measurements that we assume will be the same in both, pre and post periods. With regard to the data resolution, the methodology will be able to operate on any resolution. Typically, the "hour" would be a reasonable time scale for data collection.

$\boldsymbol{E}_{[\text {pre,i] }}$, the energy consumption at a measurement time [i] of the [pre] period.

$\boldsymbol{E}_{[\text {post,i], }}$, the energy consumption at a measurement time [i] of the [post] period.

$\sum_{i=1}^{N} E_{[\text {pre, },]}$, total energy consumption in the pre-retrofit period.

$\sum_{i=1}^{N} E_{[\text {post, }]}$, total energy consumption in the post-retrofit period.

We will now define methods for adjusting this baseline figure for the three external parameters suggested above.

\subsection{Adjusting for Outdoor Temperature}

Let us consider that $\mathrm{T}_{\text {pre, }}$ and $\mathrm{T}_{\text {post,i }}$ represent the external temperature at time [i] within the [pre] and [post] periods respectively. For simplicity and with no loss of generality the resolution of the temperature data is set similar to that of energy data. In the unlikely case that $T_{\text {pre,i }}=T_{\text {post,i, }}$, for all [i], external temperature will not affect our impact figure in Equation (2). Thus, if the energy consumption before the retrofit is 100 and the respective energy consumption after the retrofit is 80 , we can safely assume that the impact of our retrofit is as shown below.

$$
\text { Impact }=[100-80] / 100=\text { an } 20 \% \text { Reduction/Saving of Energy }
$$

Obviously, if the equation $\mathrm{T}_{\text {[pre] }}=\mathrm{T}_{\text {[post] }}$ for all [i] does not apply, the above impact figure is not valid any more. The energy consumption is now affected by the different weather among the two periods.

We propose to adjust for the weather by extending a well known concept in the related literature, that of heating/cooling degree hours. Fels et al. [11] were the first to utilize a similar concept, of the variable-base degreeday in retrofitting. A similar approach was used in [12]. Here, we expand this concept by increasing its resolution at the hour level. Heating/cooling degree hours, noted as Hdh and Hch respectively are a means to describe the thermal needs of a building. Their calculation is very simple and is based on the comparison of the hourly outdoor temperature with a preset reference temperature (e.g. 20 deg). Let us see how this would work.

Let us assume two, equal in time, periods across the year, the first representing a heating period with a reference temperature set to 20 and the second a cooling period with a reference temperature of 26 degrees. A year has 8760 hours, therefore 4380 would be considered as heating hours and an equal 4380 as cooling hours. Any other separation between heating and cooling hours is possible, without any loss of generality. Even three or four periods can be easily accommodated in the methodology.

For every hour of our temperature monitoring during the heating period we would subtract the measured temperature from the reference temperature and calculate the degree hours during that, particular, hour. Following the same rule, across the whole heating period, we would end up with a total of heating degree hours. Expanding over to the cooling period we would calculate, in an identical way, the total of the cooling degree hours. Obviously, the harsher the winter, the higher the heating degree hours; the hotter the summer, the higher the cooling degree hours; and vice versa.

In this way, we have captured in a single metric the impact of weather. Obviously, this is a linear approximation and, because of this, introduces some error. A linear approximation essentially implies that the heating energy required to raise, as an example, the indoor temperature one degree when the outdoor temperature is 8 degrees is the same as that required to raise the indoor temperature one degree when the outdoor temperature is 18 degrees. This, in theory is not true, however in practice it is close to being true. The linear approximation proposed is a workable methodology, easy to implement. Any more sophisticated approach would introduce complex phenomena that could perhaps increase the accuracy to, however, a large and unacceptable loss of practicality. 
Let us know see an example to see how our impact is adjusted against the outdoor temperature. We will use the same as above example, where the energy consumption before the retrofit is 100 and the respective energy consumption after the retrofit is 80 . However, now the equation $\mathrm{T}_{\text {pre, } \mathrm{i}}=\mathrm{T}_{\text {post, } \mathrm{i}}$ for all [i], does not apply any more. In addition, we have carried out our real time calculation of heating degree hours for both periods, before and after the retrofit. Thus, let us say the $\mathrm{Hdh}_{\text {pre }}=1500$ and the $\mathrm{Hdh}_{\text {post }}=2000$.

The figures above indicate that in the period after the retrofit, the weather has been much harsher as reflected in the significantly higher number of heating degree hours. Our linear model would thus require an offset factor as follows:

$$
\text { Weather Adjustment Factor }=\mathrm{Hdh}_{\text {post }} / \mathrm{Hdh}_{\text {pre }}=2000 / 1500=4 / 3
$$

Therefore our impact figure, adjusted for weather, denoted by impact [w], would now assume the following value:

$$
\text { Impact }[w]=\text { Baseline Impact Weather Adjustement Factor }=20 \% 4 / 3=26.7 \%
$$

One may now ask whether we need to carry out a similar exercise during the cooling period. A closer look to the retrofit is required to answer this point. If, for example, the retrofit includes elements (e.g. window changes) that affect their $\mathrm{g}$ value, monitoring during the cooling period would be necessary as changes of $\mathrm{g}$ values will manifest more strongly during the summer period.

\subsection{Adjusting for Indoor Comfort}

This purpose of this adjustment is to offset any different indoor comfort conditions between the [pre] and [post] periods and to dissociate them from the true retrofit impact. Different indoor conditions may, for example, result because of a different thermostat setting, between the two periods. If users, for any reason, opt for a different indoor temperature before or after the retrofit, this will affect energy consumption figures. To isolate the true retrofit impact one would need to carry out an adjustment, along a similar, as above, rationale. Instead of "heating degree hours" we will now use the concept of "comfort degree hours", noted as Cdh. Comfort degree hours are calculated by subtracting the indoor temperature from a reference comfort temperature set, for example at 22 degrees. The calculation is done is an exactly similar way. Real time indoor, now, temperatures are subtracted every [i] time from this reference temperature to calculate the comfort hours. We would again opt for a linear model, for the same reasons as in the case of weather. Also, we restrict to the temperature aspect of indoor climate and do not consider humidity changes. However, an enthalpy, instead of a temperature, adjustment would be perfectly possible to include also humidity changes in the adjustment factor. That would not increase at all the cost or the complexity of the exercise, especially as humidity sensors are of a low cost and often come together with ambient temperature ones.

As an example, let us assume we have calculated a figure of $\mathrm{Cdh}_{\text {pre }}=630$ and the $\mathrm{Cdh}_{\text {post }}=420$. This would imply that in the post retrofit period the indoor environment was more "cold" resulting to a much decreased value of the comfort degree hours. We would therefore need to compensate this fact and offset it from our impact calculation. Similar as above, we would establish a comfort adjustment factor as follows:

$$
\text { Comfort Adjustment Factor }=\mathrm{Cdh}_{\text {post }} / \mathrm{Cdh}_{\text {pre }}=420 / 630=2 / 3
$$

One can notice that, in this case, the impact of the different comfort conditions has a negative impact on true impact (factor 2/3). Our new impact indicator, adjusted also for indoor comfort, denoted by impact [w, c], would now be:

$$
\text { Impact [w, c] }=\text { Impact }[\mathrm{w}] \text { Comfort Adjustement Factor }=26.7 \% \text { 2/3 }=17.8 \%
$$

\subsection{Adjusting for Occupancy}

Occupant densities in office buildings can vary between $4.3 \mathrm{~m}^{2}$ and $22.8 \mathrm{~m}^{2}$ per person, and this range obviously significantly impacts internal heat gains [13] [14]. Overall, it is now widely acknowledged in the literature [15][25] that occupant behavior plays a major role in determining building energy use. It is usually the main reason causing the significant gaps between actual and predicted energy performance [16] [26] [27] of buildings. Studies have shown that occupant behavior may vary to such an extent that the resultant building energy use may differ by a factor of two or more! [28] [29]. 
Building occupancy profiles may change for a number of reasons. For example, the working hours may change. The spaces may change, may increase or temporarily decrease due to maintenance works. Then the number of people may increase, decrease or change across time and space. Unpredictable events, natural catastrophes, strikes, may further add to the occupancy changes.

Do occupancy changes affect our energy considerations? The case deserves here some special attention, as it is not as straightforward as in the above cases, for indoor and outdoor conditions.

Let us imagine an office that accommodates ten office employees and in the post retrofit period their number has increased to twelve. Obviously the energy efficiency (energy consumed per person) of the space has decreased but the energy requirements of it will have remained more or less the same. In this case it would not really make sense to compensate for the new occupancy. On the other hand if, as a result of the retrofit itself, new spaces are made operational or old ones are decommissioned then this occupancy change would have an impact also on the energy profiles. Thus, there are two broad cases for occupancy.

Occupancy changes during retrofits may be due to the change of the effective building area or the timing of its use. In a way, our reference building after the retrofit has now somehow changed; this will result to a different energy consumption profile. Thus, there is a need to account for the different occupancy before and after the retrofit.

Occupancy changes may also be due to changing space use profiles, as in the example above where people office density increased. In this case, it does not seem appropriate to compensate for occupancy, as the energy consumption will only marginally be affected by such changes.

However, in the case of the occupancy, it might not be the best possible approach to keep on looking into the retrofit impact in terms of changes of the energy consumption per year (KWH/year). Indicators that consider space efficiency rather than consumption (KWH/person/year) seem much better positioned to model the true occupancy retrofit impact. Indeed, such indicators would now be affected by our "density" like, occupancy, changes.

This discussion highlights a different type of retrofitting; operational retrofitting, i.e., changing our buildings' use profiles so that they may serve more people with the same energy, so that they may be more use efficient. At the end, "energy per serviced user-hours" would be the ultimate building energy efficiency indicator. It would be far more comprehensive than the usual "energy per hypothetical population" or "energy per footage”.

As a summary, we have highlighted above that the issue of occupancy holds an important potential. However, its treatment is not as straightforward as its heating and comfort degree hours peers. The analysis will be different depending on whether we are looking at the retrofit impact on energy consumption or if we are also interested in the more subtle space performance, in the case where our retrofit has included aspects of operational changes or retrofits.

Let us consider these two cases independently.

The classical retrofit and the case of building/space energy demand.

In this case, we assume our retrofit has had by design no intention to affect directly or indirectly the operational profiles of the space/ building. Here we need to investigate, one by one, the occupancy changes that may have resulted. Typical issues to consider here are:

Are new building parts put in operation or are older ones decommissioned? Has their use timing changed?

Such issues will have an impact on the energy consumption. An occupancy based adjustment will be required.

The operational retrofit and the case of building/space energy performance.

In this case, we assume our retrofit has had by design an intention to affect directly or indirectly the operational profiles of the space/building. We are now entitled to have these, now increased, occupancies, incorporated in our impact indicator in a favorable way.

Our adjustment approach will be similar to the two above discussed adjustments, for external and internal temperature. The adjusting parameter will now be the average, headcount, occupancy, denoted in the following as Occ.

Let us assume we have calculated a figure of $\mathrm{Occ}_{\mathrm{pre}}=30$ and the $\mathrm{Occ}_{\mathrm{post}}=40$. This would imply that in the post retrofit period more people are serviced, either because of the retrofit design or for any other reason as those discussed above (e.g., an increase or the operational building hours). Again, we would now need to compensate this fact and offset it from our impact calculation. Our occupancy adjustment factor would now be cal- 
culated as follows:

$$
\text { Occupancy Adjustment Factor }=\text { Occ }_{\text {post }} / \text { Occ }_{\text {pre }}=40 / 30=4 / 3
$$

Our new impact indicator, adjusted also for occupancy and denoted by impact [w, c, o], would now be,

$$
\text { Impact }[\mathrm{w}, \mathrm{c}, \mathrm{o}]=\text { Impact }[\mathrm{w}, \mathrm{c}] \text { Occupancy Adjustement Factor }=17.8 \% 4 / 3=23.7 \%
$$

\section{Conclusions}

We have presented above a conceptual framework for managing and carrying out adjustments when trying to assess the impact of a retrofit. We have suggested that these adjustments should include compensation for different outdoor (change of outdoor temperature), different indoor (change of indoor temperature), changes in building occupancy and use profiles.

Besides emphasizing the need to carry out adjustments along the three above parameters, we have developed a concrete methodology for carrying them out. In the case of indoor and outdoor conditions, the methodology is an extension of the well-known concept of heating days/hours. Heating/cooling hours will offset the weather impact while comfort hours will adjust the indoor climate variation. In this paper, we have modeled the comfort hours exclusively on indoor temperature; this can be readily extended to an enthalpy based indicator, including also the humidity changes.

In the case of occupancy, we have considered it necessary to differentiate between occupancy changes that come as a result of the retrofit itself and those resulting in an ad hoc manner. In the first case we have a so called operational retrofit; this should be always accounted for in the retrofit impact calculation. An operational retrofit reconsiders the space use and space user deployment in view of a better energy performance. Operational retrofits hold some important savings' potential and have been somehow overlooked as to their potential to deliver energy savings. However, the way that a building is operated can have an important impact, maybe comparable to the way that it is insulated. This is especially relevant in the case of new and modern building designs that have already introduced advanced insulation technologies and have rather little to expect from enhancements of their shell insulation or energy systems.

The ability to measure occupancy (headcount) in a cost efficient way would add to the proliferation and validation of such concepts. This appears to be quite a serious limitation to the practical uptake and validation of operational retrofits.

In the case of occupancy, besides operational retrofits, one needs to also consider all other ad hoc occupancy changes, on a case per case basis, in order to see if and how they may affect the retrofit impact and should, therefore, be uptake in the adjustment methodology. For example, the change of the timing of the building use and the effective building area would also require adjustment.

\section{Discussion-Further Research}

The work presented here is a conceptual part of a larger ongoing research aiming at practical retrofit evaluation. The adjustment methodology presented above will be incorporated there together within another major topic: the definition of the consumption, baseline and post retrofit, in building spaces. Whenever our retrofit scope is that of a building space alone (a floor, a set of rooms, etc.), the measurement of the HVAC energy consumed in that space may be a very hard exercise indeed. Similar may apply if we want to look into a specific part of the retrofit strategy that has been applied to a specific part of the building. We will now need a complementary concept to manage such cases; otherwise the applicability of the adjustment methodology will be limited. In addition, the monitoring protocol duration is a highly important, issue. The more we can shorten it and still reach valid impact results, the more promising the methodology would be in true business environments.

Field validation will be required for the full methodology, energy baseline and post retrofit definition and adjustment incorporation, to monitor how the calculated impact evolves as the monitoring duration increases. We hope in this way to define an "effective duration", required for this exercise to converge a safe assessment of the impact. We also expect this to be close to that of a week.

In short, the next step of this research would be a full and comprehensive methodology for retrofit evaluation that would be practical and short in time, without the requirement to resort to costly and expensive measurements (e.g. velocities in air and ducts, etc.) and without the need to carry on the campaign for long pe- 
riods. These two conditions would grant the methodology some significant opportunity for true industry uptake.

\section{Acknowledgements}

The work presented in this paper is partly funded by the European Commission within the 7th Framework Programme (RESSEEPE Project, Grant Agreement No: 609377).

\section{References}

[1] IPMVP, International Performance Measurement and Verification Protocol (2010) Concepts and Options for Determining Energy and Water Savings. Efficiency Valuation Organization.

[2] ASHRAE (2002) Ashrae Guideline 14: Measurement of Energy and Demand Savings. American Society of Heating, Refrigerating, and Air-Conditioning Engineers Inc., Atlanta, GA.

[3] Burkharta, M.C., Heob, Y. and Zavala, V.M. (2014) Measurement and Verification of Building Systems under Uncertain Data: A Gaussian Process Modeling Approach. Energy and Buildings, 75, 189-198. http://dx.doi.org/10.1016/j.enbuild.2014.01.048

[4] Srivastava, A., Tewaria, A. and Dongb, B. (2013) Baseline Building Energy Modeling and Localized Uncertainty Quantification Using Gaussian Mixture Models. Energy and Buildings, 65, 438-447. http://dx.doi.org/10.1016/j.enbuild.2013.05.037

[5] Wang, L.P., Mathew, P. and Pang, X.F. (2012) Uncertainties in Energy Consumption Introduced by Building Operations and Weather for a Medium-Size Office Building. Energy and Buildings, 53, 152-158. http://dx.doi.org/10.1016/j.enbuild.2012.06.017

[6] Walter, T., Price, P.N. and Sohn, M.D. (2014) Uncertainty Estimation Improves Energy Measurement and Verification Procedures. Applied Energy, 130, 230-236. http://dx.doi.org/10.1016/j.apenergy.2014.05.030

[7] Kissock, J.K. (1993) A Methodology to Measure Retrofit Energy Savings in Commercial Buildings. Ph.D. Thesis, Department of Mechanical Engineering, Texas A\&M University.

[8] Krarti, M., Kreider, J., Cohen, D. and Curtiss, P. (1998) Prediction of Energy Saving for Building Retrofits Using Neural Networks. Journal of Solar Energy Engineering, 120, 47-53. http://dx.doi.org/10.1115/1.2888071

[9] Dhar, A., Reddy, T. and Claridge, D. (1999) A Fourier Series Model to Predict Hourly Heating and Cooling Energy Use in Commercial Buildings with Outdoor Temperature as the Only Weather Variable. Journal of Solar Energy Engineering, 121, 47-53. http://dx.doi.org/10.1115/1.2888142

[10] Reddy, T.A., Saman, N.F., Claridge, D.E., Haberl, J.S., Turner, W.D. and Chalifoux, A.T. (1997) Baselining Methodology for Facility-Level Monthly Energy Use-Part 1: Theoretical Aspects. ASHRAE Transactions, 103, Part 2.

[11] Fels, M. (1986) Special Issue Devoted to Measuring Energy Savings, the Princeton Score Keeping Method (PRISM). Energy and Buildings, 9, 5-18.

[12] Kaiser, M.J. and Pulsipher, A.G. (2010) Preliminary Assessment of the Louisiana Home Energy Rebate Offer Program Using IPMVP Guidelines. Applied Energy, 87, 691-702. http://dx.doi.org/10.1016/j.apenergy.2009.08.001

[13] Knight, I.P. and Dunn, G.N. (2003) Evaluation of Heat Gains in UK Office Environments. Worldwide CIBSE/ASHRAE Gathering of the Building Services Industry, Edinburgh.

[14] Hoes, P., Hensen, J.L.M., Loomans, M.G.L.C., de Vries, B. and Bourgeois, D. (2009) User Behavior in Whole Building Simulation. Energy and Buildings, 41, 295-302. http://dx.doi.org/10.1016/j.enbuild.2008.09.008

[15] Seligman, C., Darley, J.M. and Becker, L. (1997) Behavioral Approaches to Residential Energy Conservation. Energy and Buildings, 1, 325-337. http://dx.doi.org/10.1016/0378-7788(78)90012-9

[16] Stern, P.C. (1985) Energy Efficiency in Buildings: Behavioral Issues. National Academy Press, Washington DC.

[17] Stern, P.C. (2000) New Environmental Theories: Towards a Coherent Theory of Environmentally Significant Behavior. Journal of Social Issues, 56, 407-424. http://dx.doi.org/10.1111/0022-4537.00175

[18] Owens, J. and Wilhite, H. (1988) Household Energy Behavior in Nordic Countries: An Unrealized Energy Saving Potential. Energy, 13, 853-859. http://dx.doi.org/10.1016/0360-5442(88)90050-3

[19] Lutzenhiser, L. (1993) Social and Behavioral Aspects of Energy Use. Annual Review of Energy and the Environment, 18, 247-289. http://dx.doi.org/10.1146/annurev.eg.18.110193.001335

[20] Mullaly, C. (1998) Home Energy Use Behavior: A Necessary Component of Successful Local Government Home Energy Conservation Programs. Energy Policy, 26, 1041-1052. http://dx.doi.org/10.1016/S0301-4215(98)00046-9

[21] Steg, L. (2008) Promoting Household Energy Conservation. Energy Policy, 36, 4449-4453. http://dx.doi.org/10.1016/j.enpol.2008.09.027 
[22] Guerra-Santin, O., Itard, L. and Visscher, H. (2009) The Effect of Occupancy and Building Characteristics on Energy Use for Space and Water Heating in Dutch Residential Stock. Energy and Buildings, 41, 1223-1232. http://dx.doi.org/10.1016/j.enbuild.2009.07.002

[23] Guerra-Santin, O. and Itard, L. (2010) Occupants’ Behavior: Determinants and Effects on Residential Heating Consumption. Building Research and Information, 38, 318-338. http://dx.doi.org/10.1080/09613211003661074

[24] Gram-Hansen, K. (2010) Residential Heat Comfort Practices: Understanding Users. Building Research and Information, 38, 175-186. http://dx.doi.org/10.1080/09613210903541527

[25] Gram-Hansen, K. (2011) Households’ Energy Use-Which Is the More Important: Efficient Technologies or User Practices. Proceedings of the World Renewable Energy Congress, Linkoping, 8-13 May 2011.

[26] Haas, R. and Biermayr, P. (2000) The Rebound Effect for Space Heating-Empirical Evidence from Austria. Energy Policy, 28, 403-410. http://dx.doi.org/10.1016/S0301-4215(00)00023-9

[27] Sunikka-Blank, M. and Galvin, R. (2012) Introducing the Prebound Effect: The Gap between Performance and Actual Energy Consumption. Building Research and Information, 40, 260-273. http://dx.doi.org/10.1080/09613218.2012.690952

[28] Baker, N. and Steemers, K. (2000) Energy and Environment in Architecture: A Technical Design Guide. Taylor \& Francis, London.

[29] Steemers, K. and Yun, G.Y. (2009) Household Energy Consumption: A Study of the Role of Occupants. Building Research and Information, 37, 625-637. http://dx.doi.org/10.1080/09613210903186661 
Scientific Research Publishing (SCIRP) is one of the largest Open Access journal publishers. It is currently publishing more than 200 open access, online, peer-reviewed journals covering a wide range of academic disciplines. SCIRP serves the worldwide academic communities and contributes to the progress and application of science with its publication.

Other selected journals from SCIRP are listed as below. Submit your manuscript to us via either submit@scirp.org or Online Submission Portal.
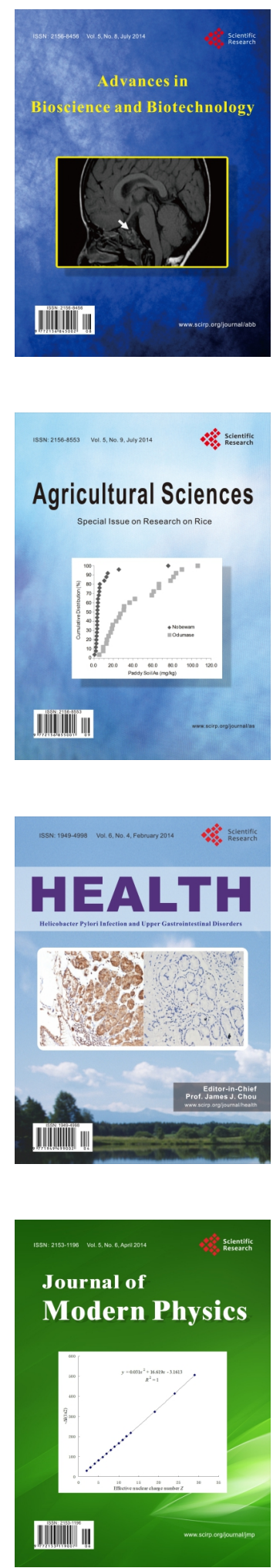
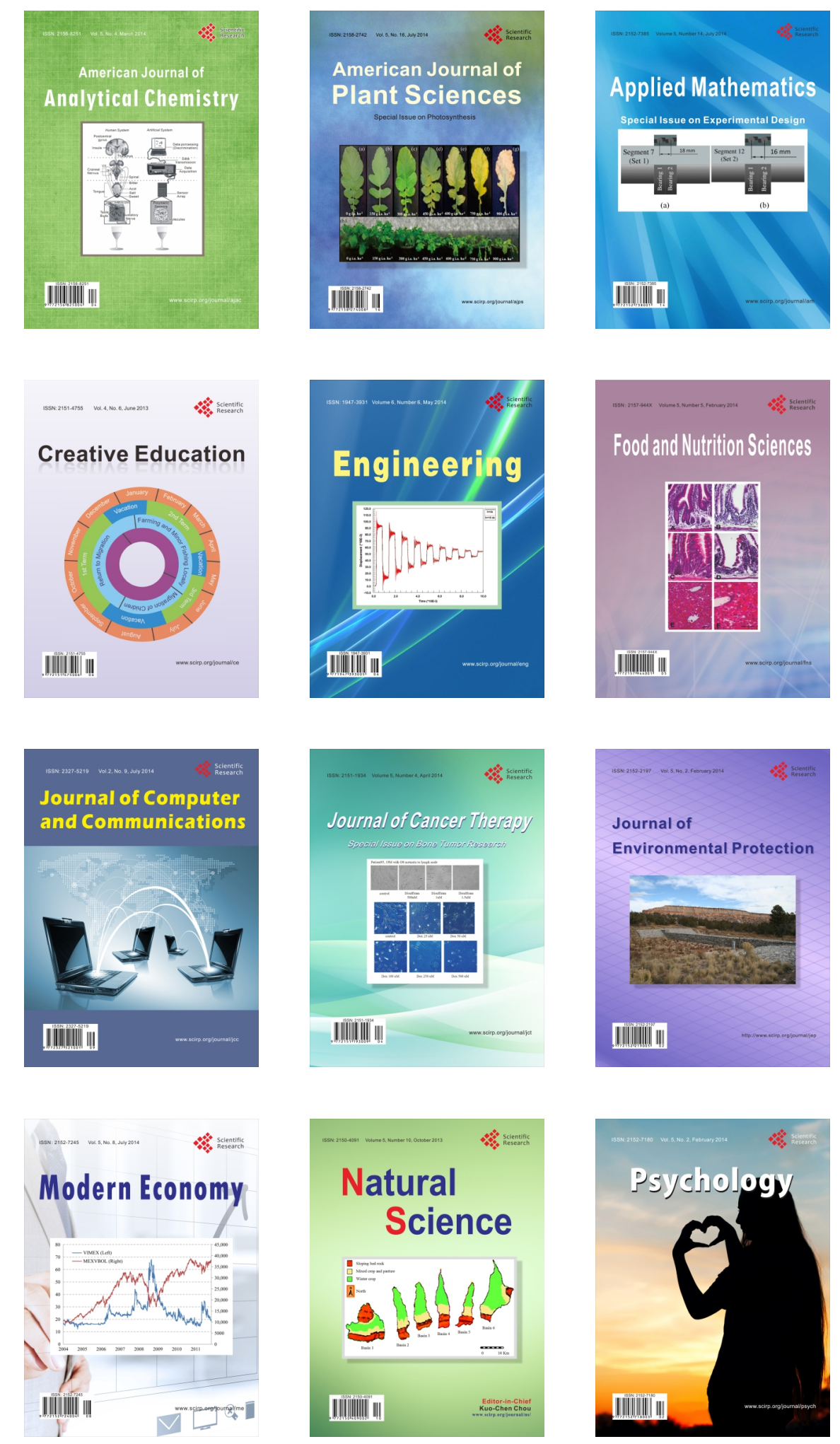